\title{
Dietary fibres reduce blood pressure, serum total cholesterol and platelet aggregation in rats
}

\author{
BY M. BAGGER', O. ANDERSEN ${ }^{2}$, J. B. NIELSEN ${ }^{1}$ AND K. R. RYTTIG \\ ${ }^{1}$ Department of Environmental Medicine, Odense University, Winslowparken 17, 5000 Odense C, \\ Denmark \\ ${ }^{2}$ Institute for Chemistry and Life Sciences, Roskilde University Centre, Box 260, 4000 Roskilde, \\ Denmark \\ ${ }^{3}$ MEDDOC, Agern Alle 15, 2970 Horsholm, Denmark
}

(Received 23 August 1994 - Revised 9 May 1995 - Accepted 27 June 1995)

\begin{abstract}
The effects of dietary fat and dietary fibres on blood pressure, serum lipids and platelet aggregation in spontaneously hypertensive and Wistar-Kyoto rats have been investigated. The systolic and diastolic blood pressure values were increased with increasing amounts of dietary fat and normalized by dietary fibre supplementation. The greatest reduction in blood pressure was obtained by a combination of reduced dietary fat and supplementary dietary fibre. Addition of dietary fibre when the amount of dietary fat was high or reduction of dietary fat when the amount of dietary fibre was low gave a smaller effect. In both rat strains the decreases in systolic and diastolic blood pressure values after reducing dietary fat and/or increasing dietary fibre were about $10-15 \mathrm{mmHg}$. Serum total cholesterol and serum HDLcholesterol concentrations were reduced by reduction of dietary fat or a combination of dietary fat reduction and dietary fibre addition. A combination of dietary fat reduction and dietary fibre addition was the most effective dietary change for reducing serum triacylglycerol concentration and platelet aggregation. The present study demonstrates that in this experimental model it is possible to reduce risk indicators of coronary heart disease significantly by changing dietary habits.
\end{abstract}

Dietary fibre: Blood pressure: Serum lipids: Platelet aggregation

Coronary heart disease (CHD) remains a leading cause of death in Western countries; therefore, prevention of CHD should be given a high priority in health care. Because CHD develops slowly without any observable sign of disease, it is important to evaluate possible risk indicators which can be identified before clinical signs of disease arise. As several of these risk indicators are influenced by lifestyle, it might be possible to postpone or prevent the development of CHD by only small changes in lifestyle. Only by postponing or preventing the disease can the goal of a sizeable reduction in the prevalence of CHD be achieved.

The most important clinical risk indicators for CHD are hypertension, increased serum total cholesterol (Se-T-Chol), increased serum levels of low-density-lipoprotein cholesterol (Se-LDL-Chol), and decreased serum levels of high-density-lipoprotein cholesterol (SeHDL-Chol). Direct intervention trials have produced evidence that these risk indicators are all influenced by the diet, and that reducing these risk indicators reduces the risk of developing CHD (Hjermann et al. 1981; Castelli, 1984; Lipid Research Clinics Program, 1984). Dietary modification therefore ought to be the first line of preventive strategy towards development of CHD. Over the past few years the influence of the diet on hypertension and hypercholesterolaemia has been discussed. The most important dietary factors are type and amount of fat. However, there has also been considerable interest in 
the role of dietary fibre as a dietary adjunct to a low-fat diet or simply as a means of reducing the energy and/or fat intake for the management of hypertension and hypercholesterolaemia.

The effect of polyunsaturated fatty acids (PUFA) on the blood pressure in spontaneously hypertensive ( $\mathrm{SH})$ rats and Wistar-Kyoto (WKY) rats is not well established. In some experimental studies in rats the addition of PUFA to the diet did not affect the blood pressure (Singer et al. 1986; Watanabe et al. 1989), whereas in others, PUFA reduced the blood pressure (Soma et al. 1985; Hoffmann et al. 1986; Singer et al. 1990). This difference could be explained by the fact that individual PUFA have different effects on the blood pressure. The effect depends on their chemical structure. Thus, dietary $n-6$ PUFA are most effective in delaying the development of hypertension, whereas dietary $n-3$ PUFA are most effective in maintaining a low blood pressure (Singer et al. 1990). Finally, a combination of different PUFA is not more effective in reducing blood pressure than the most effective PUFA alone (Singer et al. 1986, 1990). PUFA also reduce Se-T-Chol and SeLDL-Chol, but there is disagreement about the effect of PUFA on Se-HDL-Chol (Goodnight et al. 1982). It has also been indicated that monounsaturated fatty acids (MUFA) have a lipid-lowering effect equal to that of PUFA (Grundy, 1986).

The role of dietary fibre with regard to blood pressure is also contradictory. In some human studies dietary fibre reduced blood pressure (Anderson, 1983; Ryttig, 1990), whereas in other studies it did not (Little et al. 1990). However, it is difficult to compare studies where different types of dietary fibre have been used, since the different types have different lipid-altering effects. In particular the water-soluble fraction of dietary fibre has a lipid-lowering effect (Behall, 1990).

Increased aggregation of platelets has also been positively related to development of CHD (Elwood et al. 1990). The diet influences the lipid composition of platelets and thus affects the function of platelets (Rand et al. 1986).

The present study differs from previous investigations in that it was designed to evaluate systematically all possible combinations of high/low-fat and high/low-fibre diets on risk indicators for developing CHD such as blood pressure, serum lipids, and platelet aggregation in a rat model. Two different experiments were carried out to see if PUFA and dietary fibre can decrease the risk indicators for developing CHD not only in hypertensive but also in normotensive rats. WKY rats were chosen as the normotensive strain and SH rats as the hypertensive strain because the SH rat is a widely used animal model considered to resemble essential hypertension in humans. In WKY rats we evaluated whether an increased amount of dietary fat would increase the risk indicators for CHD and whether the possible increase could be normalized again by adding fibre to the feed. In $\mathrm{SH}$ rats, all combinations of high/low dietary fat and high/low dietary fibre were evaluated for their ability to change an already increased level of the risk indicators for CHD. The possible combinations of high/low dietary fat and high/low dietary fibre were used to separate the effects arising from low dietary fat and the effects arising from the high dietary fibre and to investigate whether a combination of both would give a stronger effect than the most effective alone.

\section{MATERIALS AND METHODS}

Animals and feeding procedure

Seventy-two male rats of the SH strain SHR/BM and fifty male rats of the normotensive WKY strain (Møllegaard Breeding Centre, Ll Skensved, Denmark) were housed in cages of five or six rats. All animals were weighed before allocation to groups to ensure weight homogeneity in each group and then marked. The environment was well controlled with a $12 \mathrm{~h}$ light-dark cycle $\left(0.5 \mathrm{~h}\right.$ twilight), a temperature of $21 \pm 1^{\circ}$, a relative humidity of 
$50 \pm 5 \%$ and 20 air changes/h. Animals had free access to water and semi-purified powdered diets prepared from well-defined components. All diets provided sufficient amounts of vitamins, minerals, essential amino acids and lipids. Lipid-soluble vitamins in $2.5 \mathrm{ml}$ soyabean oil were added to all diets as part of the lipid component. Cellulose $(20 \mathrm{~g} / \mathrm{kg}$, beechwood; Frisenette, Ebeltoft, Denmark) was used as the dietary fibre source in diets 1, 2 and 3, whereas a mixture of Fibradan 20 (Axel Toft, Roslev, Denmark; pea fibre) and Fibrex 610 (Fibrex AB, Malmø, Sweden; sugarbeet fibre) $(2: 1 \mathrm{w} / \mathrm{w} ; 300 \mathrm{~g} / \mathrm{kg}$ diet) was used in diets 4 and $5(660 \mathrm{~g}$ water-insoluble dietary fibre $/ \mathrm{kg}$ and $110 \mathrm{~g}$ watersoluble dietary fibre $/ \mathrm{kg}$ ). To assure homogeneous mixing of the diet, ingredients present in small amounts (oil, vitamins, minerals, cellulose) were initially mixed, and protein, carbohydrates and dietary fibre were then added. Diet components were: maize starch, potato starch and yellow dextrin (Apodan, Copenhagen, Denmark), sodium caseinate (DMV, Vejle, Denmark), soyabean oil (Aarhus Oliemølle, Aarhus, Denmark), cod-liver oil (Omekol, Nycomed dak, Copenhagen, Denmark), coconut oil (alfa-solo A/S, Vejen, Denmark), minerals and vitamins (Levnedsmiddelstyrelsen, Copenhagen, Denmark), cellulose and the dietary fibre mixture. The energy value of each feed component was used to calculate the composition of the various diets necessary to obtain the desired fractional energy contribution from each major diet component. Minerals and vitamins were added to the diets on a weight basis and not on the basis of energy density. The energy value of the dietary fibre was not included in the total energy value of the diets. The compositions of the different diets are given in Table 1.

At the age of 12 weeks the rats were offered semi-purified diets (all but one group on diet 3). After 21 weeks (Expt 1) or 43 weeks (Expt 2) diets were, except for the controls, changed according to Table 2 .

\section{Measurement of blood pressure and pulse}

The systolic blood pressure (SBP), the diastolic blood pressure (DBP) and the pulse rate were measured in the morning either weekly (Expt 1) or every fortnight (Expt 2) by indirect blood-pressure measurements (Digital Pressure Meter, LE 5000, Letica, Barcelona, Spain). The rats were placed in a pre-warmed restraining apparatus and warmed in a hot box at $35^{\circ}$ for a short time. A cuff was placed proximally on the rat's tail and a transducer, which detects the pulsation, was placed distally. When the pressure in the cuff rises there is a gradual closing of the blood flow in the tail. When pulsation stops, the pressure of the system equals SBP. When the pressure of the system falls the pulsation gradually returns to normal. When the pulsation reaches the normal rate the pressure of the system is defined as being equal to DBP. In Expt 1, animals were 24 weeks of age when measurements were started and 49 weeks of age when the experiment was terminated. For Expt 2 the animals were 48 weeks of age when measurements started and 70 weeks of age when they ended. For each determination, ten consecutive measurements of pressure and pulse of one animal were performed and the median value calculated. Thus, pressure and pulse values representing one group are medians of the medians from each animal in the group. To evaluate changes in pressure and pulse during the experiments, start and terminal values were calculated for each group, using only the last 7-9 weeks of the initial period and the last 5 weeks of the last period. In the two periods the medians of pressure and pulse were calculated for each animal for the period and the median of these medians was used for each group. 
Table 1. Contents of protein, lipid and carbohydrate (\% total energy), dietary fibre $(\mathrm{g} / \mathrm{kg}$ diet) and total energy $(\mathrm{kJ} / \mathrm{kg}$ diet $)$ in the experimental diets*

\begin{tabular}{lrrrrrr}
\hline \hline Diet... & 1 & 2 & 3 & 4 & 5 \\
\hline Protein (\% total energy) & 15 & 20 & 20 & 20 & 20 \\
Lipid (\% total energy) & 10 & 20 & 50 & 50 & 20 \\
Carbohydrate (\% total energy) & 75 & 60 & 30 & 30 & 60 & 300 \\
Dietary fibre (g/kg) & 20 & 20 & 20 & 300 & 12500 \\
Total energy (kJ/kg) & 16800 & 17800 & 21900 & 15300 & 12500 \\
\hline
\end{tabular}

* In all diets the protein source was casein. In diet 1 the source of lipid was cod-liver oil, in diets 2 and 5 it was a mixture of coconut oil and soyabean oil, and in diets 3 and 4 it was coconut oil. In diets 1,2 and 3 the source of dietary fibre was cellulose whereas in diets 4 and 5 it was a mixture of Fibradan 20 (pea fibre) and Fibrex 610 (sugarbeet fibre) $(2: 1, \mathrm{w} / \mathrm{w})$.

Table 2. Design of the dietary regimens for experiments 1 and $2^{*}$

\begin{tabular}{lcc}
\hline Expt 1: WKY & Diet at 12 weeks of age & Diet at 33 weeks of age \\
Group 1 $(n 15)$ & 1 & 1 \\
Group 2 $(n$ 20) & 3 & 4 \\
Group 3 $(n$ 15) & 3 & 3 \\
Expt 2: SH & Diet at 12 weeks of age & Diet at 55 weeks of age \\
Group 1 $(n$ 18) & 3 & 3 \\
Group 2 $(n 18)$ & 3 & 2 \\
Group 3 $(n$ 18) & 3 & 5 \\
Group 4 $(n 18)$ & 3 & 5 \\
\hline
\end{tabular}

WKY, Wistar-Kyoto rats; $\mathrm{SH}$, spontaneously hypertensive rats.

* For details of diets, see Table 1.

\section{Measurement of serum lipids}

At the end of the study, blood was collected after an overnight fast by the method described under platelet aggregation. Blood was allowed to clot for about $2 \mathrm{~h}$ at room temperature and serum was prepared by centrifugation for $10 \mathrm{~min}$ at $3500 \mathrm{~g}$. Serum was stored at $-18^{\circ}$ for later analysis of Se-T-Chol, Se-HDL-Chol and serum triacylglycerol (Se-TG). The median of the results for each group was calculated.

\section{Measurement of platelet aggregation}

Rats were killed by withdrawing blood from the heart under mebumal ${ }^{\circledR}$ anaesthesia. All samples were collected into plastic syringes containing $1 / 10$ volume of $0.13 \mathrm{M}$-trisodium citrate ( $38 \mathrm{~g}$ trisodium citrate dihydrate/1, Merck). All samples were kept at room temperature until testing, for a period ranging from $5 \mathrm{~min}$ to $1 \mathrm{~h}$. The anticoagulated blood was mixed with an equal volume of isotonic saline solution $(9 \mathrm{~g} \mathrm{NaCl} / 1)$. Diluted citrated whole blood $(1000 \mu \mathrm{l})$ was transferred to an aggregometer cuvette placed in the thermostated $\left(37^{\circ}\right)$ cuvette holder of a whole-blood aggregometer (model 500, Chrono-log Corp., Hawertown, PA, USA). Stirring bar and electrode were added, and the system was allowed to come to equilibrium. Collagen $(4 \mu 1 ; 1 \mathrm{mg} / \mathrm{ml}$, Chrono-log Corp.) was added, 
and the change in impedance, which reflects platelet aggregation around platelets adherent to electrodes, was recorded until the maximum extent of aggregation was reached. The extent of aggregation was determined from the maximum height of response, and the rate of aggregation from the slope at the steepest part of the curve. Each blood sample was tested twice and the medians were calculated for each group.

\section{Measurement of body weight and energy intake}

Animals were weighed weekly (Expt 1) or every fortnight (Expt 2). In a period of 3 weeks (Expt 1) and 2 weeks (Expt 2) feed consumption on a group basis was measured.

\section{Statistical analysis}

As results of only two groups at a time were compared or the start and end results were compared for one group, the non-parametric Mann-Whitney test was used (level of significance chosen at $P<0 \cdot 05$, two-sided). The effects of dietary changes on SH and WKY rats were not compared.

\section{RESULTS \\ Blood pressure and pulse rate}

Expt 1. WKY rats fed on a very-low-fat, low-fibre diet maintained significantly lower blood-pressure values, both SBP and DBP, than did rats fed on a high-fat, low-fibre diet (group 1 in Table 3). However, the same low level of blood pressure was obtained by addition of dietary fibre to a high-fat diet (group 2 in Table 3). Thus, increases in both SBP and DBP induced by a high-fat, low-fibre diet (group 2 in Table 3) returned to normal after addition of dietary fibre.

Expt 2. The dietary change causing the largest effect on blood pressure in $\mathrm{SH}$ rats was the combination of reduced dietary fat and increased dietary fibre (group 4 in Table 4). A somewhat smaller effect was produced by addition of dietary fibre when the amount of dietary fat was high (group 3 in Table 4) or by reducing dietary fat when the amount of dietary fibre was low (group 2 in Table 4).

In WKY rats, addition of dietary fibre to a high-fat diet reduced the pulse rate (Table 3). In SH rats, only a combination of dietary fat reduction and addition of dietary fibre significantly reduced the pulse rate (Table 4).

\section{Serum lipids}

In WKY rats the Se-T-Chol concentration was lowest in the very-low-fat, low-fibre group, highest in the high-fat, low-fibre group, and intermediate in the high-fat, high-fibre group (Table 5). Similar tendencies were observed in the concentrations of Se-HDL-Chol and SeTG. In SH rats the concentrations of Se-T-Chol and Se-TG seemed to be lowest in the lowfat, high-fibre group, highest in the high-fat, low-fibre group and intermediate in the highfat, high-fibre and low-fat, low-fibre groups. In contrast, the concentration of Se-HDLChol was highest in the high-fat, high-fibre group, while a difference was not observed between the other three groups (Table 5).

\section{Whole-blood platelet aggregation}

The extent and rate of aggregation were highest in rats fed on a high-fat, low-fibre diet and lowest in rats fed on a low-fat or very-low-fat, low-fibre diet for both SH and WKY rats and the extent of aggregation was intermediate in WKY rats fed on a high-fat, high-fibre diet. All differences were significant (Table 6). The same tendency was seen in both extent and rate of aggregation in SH rats and in the rate of aggregation in WKY rats. 
Table 3. Expt 1. Systolic blood pressure (SBP), diastolic blood pressure $(D B P)(\mathrm{mmHg})$ and pulse rate (beats/min) of Wistar-Kyoto rats at the beginning and end of a period on an experimental dietary regimen§

(Median values with 25 th and 75 th percentiles given in parentheses)

\begin{tabular}{|c|c|c|c|}
\hline & Group $1(n 15)$ & Group $2(n 20)$ & Group $3(n 15)$ \\
\hline \multicolumn{4}{|c|}{$\mathrm{SBP}(\mathrm{mmHg})$} \\
\hline Start & $139(134-150)$ & $154 *(148-160)$ & $155^{*}(151-160)$ \\
\hline End & $133 \pm(126-135)$ & $137 \ddagger(134-141)$ & $145^{* \dagger} \ddagger(141-150)$ \\
\hline \multicolumn{4}{|c|}{$\mathrm{DBP}(\mathrm{mmHg})$} \\
\hline Start & $110(98-113)$ & $123 *(113-128)$ & $124 *(117-130)$ \\
\hline End & $104(100-109)$ & $106 \ddagger(101-112)$ & $118 * \dagger \ddagger(109-120)$ \\
\hline \multicolumn{4}{|c|}{ Pulse rate (beats/min) } \\
\hline Start & $320(308-333)$ & $336(313-360)$ & $323(307-359)$ \\
\hline End & $309(282-327)$ & $289 \ddagger(267-314)$ & $313(301-318)$ \\
\hline
\end{tabular}

* Significantly different from group 1 (Mann-Whitney, $P<0.05$ ).

+ Significantly different from group 2 (Mann-Whitney, $P<0.05$ ).

\$ Significantly different from start period (Mann-Whitney, $P<0.05$ ).

$\$$ Group 1, very-low-fat, low-fibre diet (diet 1) in both periods; group 2, high-fat, low-fibre diet (diet 3) at the start and high-fat, high-fibre diet (diet 4) at the end; group 3, diet 3 in both periods. For details of diets see Table 1.

Table 4. Expt 2. Systolic blood pressure (SBP), diastolic blood pressure $(D B P)(\mathrm{mmHg})$ and pulse rate (beats/min) of spontaneously hypertensive rats at the beginning and end of a period on an experimental dietary regimen

(Median values with 25 th and 75 th percentiles given in parentheses)

\begin{tabular}{|c|c|c|c|c|}
\hline & Group 1 ( $n$ 18) & Group 2 (n 18) & Group $3(n$ 18) & Group 4 (n 18) \\
\hline \multicolumn{5}{|c|}{$\mathrm{SBP}(\mathrm{mmHg})$} \\
\hline Start & $232(223-242)$ & $229(226-235)$ & $230(226-233)$ & $227(214-238)$ \\
\hline End & $229(215-234)$ & $218 \S(210-225)$ & $2168(210-224)$ & $211 * \S(201-223)$ \\
\hline \multicolumn{5}{|c|}{ DBP (mmHg) } \\
\hline Start & $188(181-200)$ & $188(183-196)$ & $189(185-194)$ & $186(173-197)$ \\
\hline End & $188(170-191)$ & $175 \S(168-180)$ & $171 \S(165-186)$ & $170 * \S(155-181)$ \\
\hline \multicolumn{5}{|c|}{ Pulse rate (beats/min) } \\
\hline Start & $427(421-437)$ & $433(411-442)$ & $433(425-441)$ & $430(416-438)$ \\
\hline End & $433(423-444)$ & $431(418-438)$ & $436(418-442)$ & $414^{*+1} \S(396-429)$ \\
\hline
\end{tabular}

* Significantly different from group 1 (Mann-Whitney, $P<0.05$ ).

$\dagger$ Significantly different from group 2 (Mann-Whitney, $P<0.05$ ).

Significantly different from group 3 (Mann-Whitney, $P<0.05$ ).

$\$$ Significantly different from start period (Mann-Whitney, $P<0.05$ ).

II Group 1, high-fat, low-fibre diet (diet 3) for the whole experiment; group 2, diet 3 at the start and low-fat, low-fibre diet (diet 2) at the end ; group 3, diet 3 at the start and high-fat, high-fibre diet (diet 4) at the end; group 4 , diet 3 at the start and low-fat, high-fibre diet (diet 5) at the end. For details of diets, see Table 1. 
Table 5. Serum total cholesterol (Se-T-Chol), HDL-cholesterol (Se-HDL-Chol) and triacylglycerol (Se-TG) concentrations (mmol/l) in Wistar-Kyoto (WKY) and spontaneously hypertensive $(\mathrm{SH})$ rats fed on diets containing different proportions of fat and dietary fibre§ (Median values with 25th and 75th percentiles given in parentheses)

\begin{tabular}{|c|c|c|c|c|c|}
\hline & $n$ & Diet & $\begin{array}{l}\text { Se-T-Chol } \\
(\mathrm{mmol} / \mathrm{l})\end{array}$ & $\begin{array}{l}\text { Se-HDL-Chol } \\
\text { (mmol/l) }\end{array}$ & $\begin{array}{c}\mathrm{Se}-\mathrm{TG} \\
(\mathrm{mmol} / \mathrm{l})\end{array}$ \\
\hline \multicolumn{6}{|l|}{ WKY } \\
\hline Group 1 & 15 & 1 & $1 \cdot 1(1 \cdot 0-1 \cdot 3)$ & $0.94(0.90-1.06)$ & $0.36(0.28-0.71)$ \\
\hline Group 2 & 20 & 4 & $2 \cdot 0 *(1 \cdot 7-2 \cdot 1)$ & $1.61 *(1.51-1.74)$ & $0.68 *(0.54-0.89)$ \\
\hline Group 3 & 15 & 3 & $2 \cdot 2 *+(2 \cdot 1-2 \cdot 4)$ & $1.71^{*}+(1.62-1.91)$ & $0.83^{*}+(0.72-0.96)$ \\
\hline \multicolumn{6}{|l|}{ SH } \\
\hline Group 1 & 18 & 3 & $2 \cdot 4(2 \cdot 3-2 \cdot 5)$ & $1.71(1.66-1.86)$ & $0.72(0.57-0.91)$ \\
\hline Group 2 & 18 & 2 & $2 \cdot 1 *(1 \cdot 9-2 \cdot 2)$ & $1.59 \neq(1.32-1.72)$ & $0.49 *(0.41-0.57)$ \\
\hline Group 3 & 18 & 4 & $2 \cdot 2 *(2 \cdot 0-2 \cdot 3)$ & $1.86(1.69-1.96)$ & $0.56 *(0.48-0.63)$ \\
\hline Group 4 & 18 & 5 & $1 \cdot 8^{*}+(1 \cdot 0-2 \cdot 3)$ & $1.55 \div(0.98-1.79)$ & $0.42 * \pm(0.33-0.48)$ \\
\hline
\end{tabular}

* Significantly different from group 1 (Mann-Whitney, $P<0-05$ ).

$\dagger$ Significantly different from group 2 (Mann-Whitney, $P<0.05$ ).

\$ Significantly different from group 3 (Mann-Whitney, $P<0.05$ ).

$\S$ For details of diets, see Table 1.

Table 6. Extent of platelet aggregation $(\mathrm{Ohm})$ and rate of aggregation $(\mathrm{Ohm} / \mathrm{min})$ after addition of $4 \mu$ l collagen to diluted whole blood from Wistar-Kyoto $(W K Y)$ and spontaneously hypertensive $(\mathrm{SH})$ rats fed on diets containing different proportions of fat and dietary fibre*

(Median values with 25 th and 75 th percentiles given in parentheses)

\begin{tabular}{|c|c|c|c|c|}
\hline & $n$ & Diet & $\begin{array}{l}\text { Aggregation extent } \\
(\mathrm{Ohm})\end{array}$ & $\begin{array}{l}\text { Aggregation rate } \\
(\mathrm{Ohm} / \mathrm{min})\end{array}$ \\
\hline \multicolumn{5}{|l|}{ WKY } \\
\hline Group 1 & 9 & 1 & $2.50(0-4.38)$ & $0.25(0-0.35)$ \\
\hline Group 2 & 18 & 4 & $4 \cdot 38 *(2 \cdot 25-6 \cdot 13)$ & $0.48(0.25-0.65)$ \\
\hline Group 3 & 15 & 3 & $6.50 *+(4.69-7.06)$ & $0.60 *(0.41-0.83)$ \\
\hline \multicolumn{5}{|l|}{$\mathrm{SH}$} \\
\hline Group 1 & 16 & 3 & $6.99(6 \cdot 19-9.50)$ & $0.84(0.69-1.26)$ \\
\hline Group 2 & 16 & 2 & $3.97 *(2 \cdot 75-5 \cdot 69)$ & $0.47 *(0.28-0.78)$ \\
\hline Group 3 & 17 & 4 & $5.43(3.16-7.66)$ & $0.64(0-35-1.08)$ \\
\hline Group 4 & 12 & 5 & $4.94^{*}(2 \cdot 47-6.07)$ & $0.54 *(0.26-0.76)$ \\
\hline
\end{tabular}

* Significantly different from group 1 (Mann-Whitney, $P<0 \cdot 05$ ).

$\dagger$ Significantly different from group 2 (Mann-Whitney, $P<0-05$ ).

$\ddagger$ For details of diets, see Table 1 .

Furthermore, rats fed on a low-fat, high-fibre diet had a significantly lower extent and rate of aggregation than rats fed on a high-fat, low-fibre diet, but the aggregation variables seemed to be higher than for rats fed on a low-fat, low-fibre diet (Table 6).

\section{Growth rate and energy consumption}

The feed consumption by rats fed on a low-fat, low-fibre diet was higher but the dietary energy intake was lower than in rats fed on a high-fat, low-fibre diet. Addition of dietary fibre reduced the dietary energy consumed because the amount eaten was almost unaltered (Table 7). The growth rates of rats fed on a very-low-fat, low-fibre diet and of rats fed on 
Table 7. Food intake ( $\mathrm{g}$ ) and dietary energy consumption ( $k J$ by Wistar-Kyoto (WKY) rats fed for 3 weeks and spontaneously hypertensive $(\mathrm{SH})$ rats fed for 2 weeks on diets containing different proportions of fat and dietary fibre, together with body weights $(g)$ at the start and end of the experiment!

(Median values with 25 th and 75 th percentiles given in parentheses)

\begin{tabular}{|c|c|c|c|c|c|c|}
\hline & $n$ & Diet & $\begin{array}{l}\text { Food consumed } \\
\text { (g) }\end{array}$ & $\begin{array}{c}\text { Dietary energy } \\
(\mathrm{kJ})\end{array}$ & $\begin{array}{c}\text { Body wt at start } \\
\text { (g) }\end{array}$ & $\begin{array}{c}\text { Body wt at end } \\
(\mathrm{g})\end{array}$ \\
\hline \multicolumn{7}{|l|}{ WKY } \\
\hline Group 1 & 15 & 1 & 11698 & 196526 & $398(388-408)$ & $443(432-448)$ \\
\hline Group 2 & 20 & 4 & 12365 & 189184 & $417^{*}(404-432)$ & $439(420-457)$ \\
\hline Group 3 & 15 & 3 & 9595 & 210130 & $422 *(415-440)$ & $463^{*}+(448-476)$ \\
\hline \multicolumn{7}{|l|}{ SH } \\
\hline Group 1 & 18 & 3 & 10843 & 237462 & $405(389-417)$ & $392(376-418)$ \\
\hline Group 2 & 18 & 2 & 13089 & 232984 & $399(372-416)$ & $394(370-418)$ \\
\hline Group 3 & 18 & 4 & 12011 & 183768 & $405(386-417)$ & $397(383-415)$ \\
\hline Group 4 & 18 & 5 & 13677 & 170963 & $395(368-407)$ & $376(353-402)$ \\
\hline
\end{tabular}

* Significantly different from group 1 (Mann-Whitney, $P<0.05$ ).

† Significantly different from group 2 (Mann-Whitney, $P<0.05$ ).

\$For details of diets and procedures, see Tables 1 and 2 and pp. $484-487$.

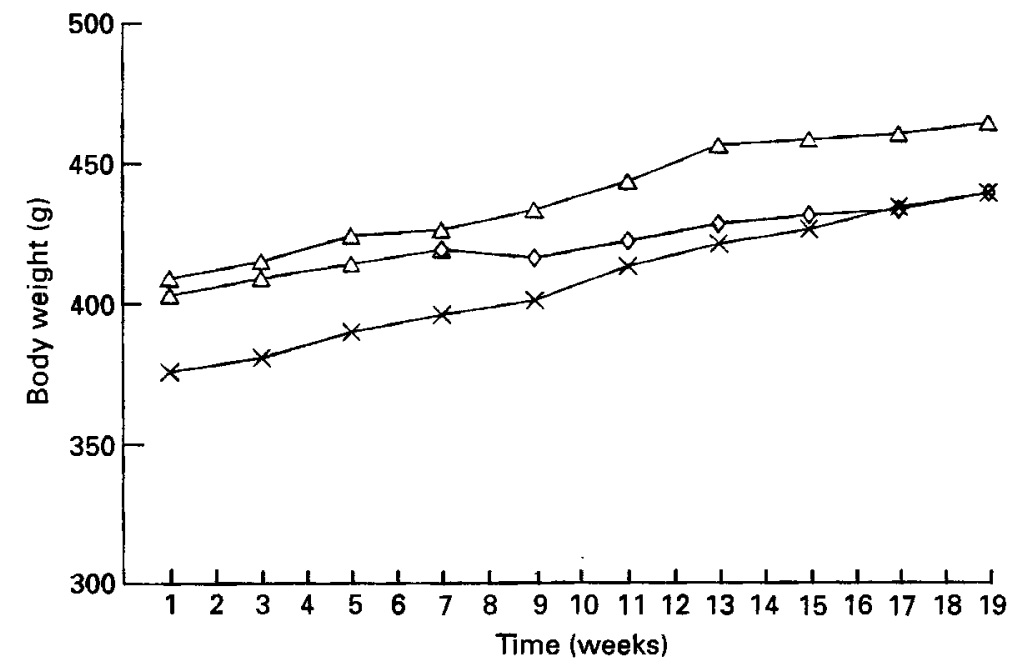

Fig. 1. Expt 1. Weight gain by Wistar-Kyoto rats fed on diets containing different proportions of fat and dietary fibre. Values are group medians. Fifteen animals received a low-fat, low-fibre diet $(x)$ for the entire experiment, twenty animals received a high-fat, low-fibre diet $(\triangle)$ for the first 7 weeks and a high-fat, high-fibre diet $(\diamond)$ for the rest of the experiment, and fifteen animals received the high-fat, low-fibre diet for the entire experiment. For details of diets see Table 1.

a high-fat, low-fibre diet were the same, but rats fed on the very-low-fat, low-fibre diet were smallest and addition of dietary fibre to the high-fat diet reduced the growth rate in rats receiving this diet (Fig. 1).

\section{DISCUSSION}

A combination of reduction of fat and addition of fibre to the diet induces the greatest reduction in blood pressure, because different reducing components are more effective 
together than the most effective component alone (Little et al. 1990). Furthermore, studies have shown that PUFA and monounsaturated fatty acids (Sundram et al. 1990) and dietary fibre (Roach et al. 1992) reduce blood lipids in rats and the effect is stronger when they are given in combination (Roach et al. 1992). The present study demonstrates that the amounts of fat and fibre in the diet affect blood pressure, Se-T-Chol, Se-TG and platelet aggregation.

One of the main results of using the present experimental model was that we were able to increase both SBP and DBP in normotensive rats by increasing the amount of dietary fat, and eventually to normalize the blood pressure again by adding fibre to the feed. Thus, the present study demonstrates a clear effect of fibre supplementation on both SBP and DBP. The key issue in this context is that not only the SBP but also the DBP is returned to normal. The majority of previously experimental studies have focused on SBP reduction, but from a pathophysiological point of view the most relevant variable is probably the DBP as this reflects the resting pressure present continually in the cardiovascular system. In both normotensive and hypertensive rats the decrease in SBP as well as in DBP after reducing dietary fat and/or increasing dietary fibre was about $10-15 \mathrm{mmHg}$. Even though this reduction is limited, a similar small reduction has been demonstrated to reduce the risk of CHD in human cohorts, as a curvilinear relationship between blood pressure and subsequent development of CHD exists (Castelli, 1984).

Further, the results of the present study demonstrate that dietary fat reduction or a combination of dietary fat reduction and dietary fibre addition lower both Se-T-Chol and Se-HDL-Chol, while addition of dietary fibre alone selectively lowers Se-T-Chol and increases Se-HDL-Chol slightly. Thus, dietary fibre offers the greatest reduction in LDL and VLDL cholesterol. Finally, a combination of dietary fat reduction and dietary fibre addition is the most effective dietary change for reducing Se-TG.

The reduction of Se-TG after addition of dietary fibre and/or reduction of dietary fat is probably due to the smaller amount of fat available in the gut for absorption and circulation in the body. The Se-TG level therefore reflects the amount of fat available in the diet.

Platelet aggregation in rats has previously been shown to be decreased after feeding PUFA (Rand et al. 1986). In the present experiments the most effective diet to lower platelet aggregation was the low-fat diet. Thus, addition of dietary fibre and reduction of dietary fat lowered the platelet aggregation more effectively than adding fibre alone but less effectively than only reducing the fat. This could be because the dietary fibre binds $n-3$ and n-6 PUFA, which are thought to enter the prostaglandin formation pathway. Dietary fibre apparently gives a natural reduction in energy consumption, despite ad lib. feeding, which is reflected by the smaller size of the animals in the groups eating diets supplemented with fibres. Animals receiving a low-fat diet also reduced the amount of energy consumed and were smaller than animals receiving a high-fat diet. Previously, a reduced amount of feed eaten was reported to lower the blood pressure in SH rats (Young et al. 1978). The energy restriction rather than the weight loss was demonstrated to be responsible for the reduced blood pressure observed in that study. Therefore, part of the effect of dietary fibre on blood pressure could be due to energy restriction.

Generally, it is difficult to compare studies where dietary fibre has been used. Even if the same dietary fibre source has been employed in different studies, the content of the different fractions may differ extensively due to different growth and preparation conditions. Furthermore, the physiological effects of combinations of dietary fibres may be very different from their effects when individual fibre types are given in purified form.

In the present experiments the amount of dietary fibre consumed was much higher than the amount eaten by most people. However, if it is primarily the water-soluble fractions, as indicated by Spiller et al. (1991) and Roach et al. (1992), that affect the risk indicators 
for CHD investigated in our experiments, the same effects could be obtained by eating a smaller amount of food containing a higher concentration of the water-soluble fibre fractions.

In conclusion, a low-fat, high-fibre diet was most effective in reducing risk indicators for CHD in the experimental model used in the present investigation. In general, a low-fat, high-fibre diet is advantageous compared with a low-fat, low-fibre diet and also, in relation to other diet-related diseases. In human weight-reduction schemes, a controlled low-fat, lowfibre diet often includes restriction of the allowed amounts eaten and thus causes fluctuations in the level of blood glucose, which quickly induces a feeling of hunger. Addition of dietary fibre to a controlled low-fat diet would restrict the ingestion of food by a natural physiological mechanism, because the fibre fills up the stomach and stabilizes the blood glucose level, inducing a feeling of satiety (Dreher, 1987). The results of the present and related experimental studies indicate that it is possible significantly to reduce risk indicators of CHD by changing the diet of the animals.

As human epidemiological studies link these risk indicators to high prevalence of CHD, and human clinical studies link dietary intervention (reduced fat or increased fibre intake) to changes in the risk indicators considered to cause a reduced risk for CHD, it seems timely to study the combined effects of increased fibre intake, reduced fat intake and increased PUFA: saturated fatty acid ratio on risk indicators for CHD in humans.

\section{REFERENCES}

Anderson, J. W. (1983). Plant fiber and blood pressure. Annals of Internal Medicine 98, 842-846.

Behall, K. M. (1990). Effect of soluble fibers on plasma lipids, glucose tolerance and mineral balance. In New Developments in Dietary Fiber, pp. 7-16 [I. Furda and C. J. Brine, editors]. New York: Plenum Press.

Castelli, W. P. (1984). Epidemiology of coronary heart disease: the Framingham study. American Journal of Medicine 76, 4-12.

Dreher, M. L. (1987). Dietary fiber and its physiological effects. In Handbook of Dietary Fiber, pp. 199-279

[O. R. Fennema, G. W. Sanderson, P. Walstra, M. Karel, S. R. Tannenbaum and J, R. Whitaker, editors]. New York: Marcel Dekker.

Elwood, P. C., Beswick, A. D., Sharp, D. S., Yarnell, J. W. G., Rogers, S. \& Renaud, S. (1990). Whole blood impedance platelet aggregometry and ischemic heart disease. Arteriosclerosis 10, 1032-1036.

Goodnight, S. H., Harris, W. S., Connor, W. E. \& Illingworth, D. R. (1982). Polyunsaturated fatty acids, hyperlipidemia and thrombosis. Review. Arteriosclerosis 2, 87-113.

Grundy, S. M. (1986). Comparison of monounsaturated fatty acids and carbohydrates for lowering plasma cholesterol. New England Journal of Medicine 314, 745-748.

Hjermann, I., Holme, I., Byre, K. V. \& Leren, P. (1981). Effect of diet and smoking intervention on the incidence of coronary heart disease. Lancet 12, 1303-1310.

Hoffmann, P., Block, H.-U., Beitz, J., Taube, C., Forster, W., Wortha, P., Singer, P. \& Naumann, E. (1986). Comparative study of the blood pressure effect of four different vegetable fats on young spontaneously hypertensive rats. Lipids 21, 733-737.

Lipid Research Clinics Program (1984). The Lipid Research Clinics Coronary Primary Prevention Trial. Journal of the American Medical Association 251, 351-374.

Little, P., Girling, G., Hasker, A., Trafford, A. \& Craven, A. (1990). A controlled trial of a low sodium, low fat, high fibre diet in treated hypertensive patients: the efficacy of multiple dietary intervention. Postgraduate Medical Journal 66, 616-621.

Rand, M. L., Hennissen, A. A. H. M. \& Hornstra, G. (1986). Effects of dietary sunflowerseed oil and marine oil on platelet membrane fluidity, arterial thrombosis, and platelet responses in rats. Atherosclerosis 62, $267-276$

Roach, P. D., Dowling, K., Balasubramaniam, S., Illman, R. J., Kambouris, A. M., Nestel, P. J. \& Topping, D. L. (1992). Fish oil and oat bran in combination effectively lower plasma cholesterol in the rat. Atherosclerosis 96, 219-226.

Ryttig, K. R. (1990). Clinical effects of dietary fibre supplements in overweight and in hypertension. PhD Thesis, Stockholm University.

Singer, P., Gerhard, U., Moritz, V., Forster, D., Berger, I. \& Heine, H. (1986). Different changes of $n-6$ and $n-3$ fatty acids in adipose tissue from spontaneously hypertensive (SHR) and normotensive rats after diets supplemented with linolenic and eicosapentaenoic acids. Prostaglandins, Leukotrienes and Medicine 24, $163-172$.

Singer, P., Moritz, V., Wirth, M., Berger, I. \& Forster, D. (1990). Blood pressure and serum lipids from SHR after 
diets supplemented with evening primrose, sunflowerseed or fish oil. Prostaglandins, Leukotrienes and Essential Fatty Acids 40, 17-20.

Soma, M., Manku, M. S. \& Horrobin, D. F. (1985). The effect of hydrogenated coconut oil, safflower oil, and evening primrose oil on development of hypertension and sodium handling in spontaneously hypertensive rats. Canadian Journal of Physiology and Pharmacology 63, 325-330.

Spiller, G. A., Farquhar, J. W., Gates, J. E. \& Nichols, S. F. (1991). Guargum and plasma cholesterol. Effect of guargum and oat fiber source on plasma lipoproteins and cholesterol in hypercholesterolemic adults. Arteriosclerosis and Thrombosis 11, 1204-1208.

Sundram, K., Khor, H. T. \& Ong, A. S. H. (1990). Effect of dietary palm oil and its fractions on rat plasma and high density lipoprotein lipids. Lipids 25, 187-193.

Watanabe, Y, Huang, Y., Simmons, V. A. \& Horrobin, D. F. (1989). The effect of dietary $n-6$ and $n-3$ polyunsaturated fatty acids on blood pressure and tissue fatty acid composition in the spontaneously hypertensive rat. Lipids 24, 638-644.

Young, J. B., Mullen, D. \& Landsberg, L. (1978). Caloric restriction lowers blood pressure in the spontaneously hypertensive rat. Metabolism 27, 1711-1714. 